\title{
JEAN-FRANÇOIS LYOTARD:
}

\section{L'AUTRE JE(U)}

"Voilà la farce que nous jouent les mots, que nous jouent les intensités et que va nous jouer d'un bout à l'autre de ce livre notre emportement même : cet emportement (...) vous atteindra rapporté, reporté, cette feuille où j'écris et qui est un instant, dans l'éblouissement et l'impatience, la peau carréssée d'une femme ou l'aplat d'une eau dans laquelle je crawle avec amour, cette feuille, vous la recevez imprimée, répétée la même, rédupliquée, vous recevez une feuille d'enregistrement (...) Croyez-vous que la morne constatation de ce différer de l'écriture nous consterne et déprime? Elle nous intéresse vivement et nous fait rebondir. S'il est un secret, c'est celui-ci, le sien :comment l'impossible juxtaposition de singularités intenses donne-t-elle lieu au registre et à l'enregistrement? Comment le différer-déplacement hors lieu-temps de la singularité d'affect donne-t-il lieu et temps à la multiplicité, puis à la généralité, puis à l'universalité, dans le concept, dans le pris-ensemble du registre, donne-t-il lieu-temps au différer-composition ou complacement? Comment la puissance donne-t-elle place au pouvoir? Comment l'affirmation fulgurante se circonscrit-elle autour d'un zéro qui en l'inscrivant la néantise et lui assigne un sens?"1.

Cette question du différer, posée au début de l'Economie libidinale, est le fond et l'origine des grands thèmes de la pensée postmoderne et pourrait être rapporté à toutes les oeuvres de Lyotard. Mais c'est $L a$ condition postmoderne, qui, semble-t-il, a été vouée à leur donner un développement et des réponses plus articulés. Devenu vite un des livres les plus discutés dans les années 80 il s'est placé au centre des débats aussi bien philosophiques que scientifiques, culturologiques, sociopolitiques et féministes. Il est vrai que l'intérêt qu'il a provoqué parmi les grands auteurs contemporains était et reste plutôt d'ordre polémique que l'expression d'un accord ou d'une admiration quelconque. Mais il n'en reste pas moins vrai que c'est lui précisément qui a rendu au mieux le fond du grand tournant du discours philosophique de la fin du XX s. le déclin de la confidence dans l'horizon de l'universalité et par-là - du Sujet.

Quelles en seraient les conséquences pour celui qui voudrait repenser ou simplement "penser la philosophie" à travers ce tournant? 
C'est cette question que je me propose de traiter ici en quelques lignes qui s'interposent de façon diachronique.

\section{Le paranoỉa de l'universel}

Le caractère essentiel des conceptions postmodernes, comme l'a bien indique Don Ihde, c'est l'aversion contre les canons, du fait que le canon en tant que tel est toujours trop général pour servir de base au curriculum particulier ${ }^{2}$. Dans La condition postmoderne cette négation ou "résistance ${ }^{n 3}$ est deployée de la premiere à la dernière page - de l'incrédulité à l'égard des métarécits au rejet du Diskurs comme recherche d'un consensus universel. L'annonciation de la FIN des grands récits pourrait paraître excessive et extrême. Mais elle n'est pas une nouvauté et n'en pretend pas l'être. La seule prétention de l'auteur, c'est de fournir une image de la condition du savoir dans les sociétés technologiquementavancées. Et ç'est là justement que Lyotard a constaté un fait réellement présent - l'éffacement des autorités. Effacement dont l'origine se situe en philosophie dans l'échec des valeurs des Lumieres, en sciences - dans la crise des fondements (l'axiomatique) de la physique, de la mécanique, des mathématiques, en l'art - dans la dissolution des "formes". En ce sens la "diagnose" de Lyotard a été préparée aussi bien historiquement qu'intellectuellement, p. ex. par des penseurs tels que Kierkegaard, Schopenhauer, Nietzsche, Marx, Freud, Wittgenstein, Heidegger, et pourrait être appelée "paranoïa de l'universel".

Sans doute les thèmes du droit du singulier et de son incommensurabilité avec l'universel et en particulier avec les totalisations hégeliennes, de l'inconsistance des constructions spéculatives, de la pluralité de la vérité, de la décentralisation du sujet universel et impersonnel de la modernité, de l'inconscient, de l'identité et de la responsabilité se trouvent déjà dans les Miettes philosophiques, Post-Scriptum, Maladie à la mort, Crainte et tremblement, Ou bien - ou bien et autres. L'intuition de Kierkegaard que la recherche de la vérité concrète, c'est la connaissance de nous-mêmes dans l'existence, $c-\grave{a}-d$ la découverte du "paroxisme de la passion" par le rejet des systèmes rationnalistes et l'application de la dialectique paradoxale ${ }^{4}$, semble réapparaître dans les assertions de Lyotard que "le projet de systeme-sujet est un échec", parce qu'il n'y a pas de métalangue universelle ${ }^{5}$. Mais le moment spécifique, c'est que chez Lyotard cet échec des discours spéculatifs à expliquer les événements réels de l'existence va de pair avec la réinterprétation du mécanisme mouvant le corps individuel et social qu'il redécouvre dans la "volonté" nietzschéenne. La 
mise en rappport de la volonté ou le désir (der Wille) avec l'Eros et la révolution permanente du délibéré (Willkür) en tant qu'abandon conscient des règles instituées est en fait la négation de la philosophie comme préscription et la restitution de ses droits de projet entierement critique $^{6}$. En ce sens l'appel de Lyotard "ouvrez le prétendu corps et déployez toutes ses surfaces"7 est une stratégie aussi bien analytique que critique. Conçue d'abord sur fond libidinal elle est étendue plus tard, dans La condition postmoderne, sur le savoir contemporain avec ses modes discursifs et textuels comme une application particuliere de la méthode généalogique de Nietzsche.

Contre l'epistème moderne privilégiant le Sujet en tant qu'autorité discursive, Nietzsche a posé dans son Wille zur Macht la question sur le sujet de l'interprétation devenue essentielle pour le postmodernisme, respectivement sur la légitimation du "vrai", du "bien", du "beau" et du "juste". Contre la formule positiviste "il n'y a que des faits", qui devraient simplement être constatés et systématisés, Nietzsche a montré qu'il n'y a pas de "faits", mais seulement des interprétations. Même des propositions de sorte que "Tout est subjectif" sont déjà une question d'interprétation. "Le sujet" - constate Nietzsche - "n'est pas quelque chose de donné, mais quelque chose d'inventé-en-addition, de mis-derrière. Mais est-il nécessaire de mettre l'interprétateur derrière l'interprétation? Cela aussi c'est une invention, une hypothèse. Dans la mesure que le concept de 'connaissance' a un sens quelconque on pourrait dire que le monde est connaissable, mais qu'il se laisse expliquer de manières différentes - il n'a pas un sens derrière soi, mais des sens innombrables..." du sujet un n'est pas nécessaire; peut-être est-il aussi possible d'admettre une pluralité de sujets (...), une sorte d'aristocratie de cellules differentes"9 devient la these dominante du postmodernisme qui met l'accent sur l'atomisation du savoir, des structures et des relations sociales. Cette thèse a eu plusieurs effets fondamentaux. Il semble que "dans cette dissémination des jeux de langage" - souligne Lyotard, "c'est le sujet social lui-même qui paraît se dissoudre. Le lien social est langagier, mais il n'est pas fait d'une unique fibre. C'est une texture où se croisent au moins deux sortes, en réalité un nombre indeterminé, de jeux de langages obéissant à des règles différentes"10. La dissolution du "sujet un" ou unique amene par-là aux questions sur le fondement de la connaissance, sur le référent des valorisations, sur les possibilités de communication et de toute justification de normes éthiques, politiques, juridiques et sociales communes. Lyotard souligne en ce sens que le savoir postmoderne exige la preuve de la légitimité des prémisses qui 
déterminent le savoir et son langage et que Nietzsche ne fait rien d'autre quand il montre que "le nihilisme européen" découle de l'application de l'exigence scientifique de vérité à elle-même ${ }^{11}$. L'érosion du principe immanent de légitimation est non seulement la raison de la crise profonde du fondationnalisme. Elle met aussi en question les traits classiques du discours scientifique et de la philosophie en tant que telle tout en exigeant la destruction du "monopole narratif" et l'introduction des "petits récits" ou récits minoritaires ${ }^{12}$, respectivement le remplacement de l'universalisme moniste par le paganisme de la différence où il n'y a ni hiérarchie, ni finalité des propositions ${ }^{13}$. Cette texture disloquée du savoir contemporain représente son paranoïa ou son état schizo inconcevable par et pour les idiomes descriptives ou préscriptives de la modernité. Sa circularité et transfiguration constantes enlevent non seulement la possibilité de rationnalisation mais elles détruisent aussi les critères de validité déjà connues et communement admis. Das Ereignis, l'événement ou l'ad-venir de la vérité, ou mieux encore la vérité s'approchant avec les pas d'oiseau (Nietzsche), la fait sinon inaccessible du moins prérogative non du cogito mais de l'empathie : "Tombons amoureux vraiment!"14 - voilà le conseil de Lyotard qui semble détenir la clé du savoir, de la compréhension et de toute communication.

Or toute empathie, aussi étrange que ça peut paraître, se détermine par le vide (emptyness) ainsi que le son par le silence. D'où le statut "ontologique" ou le fond de l'événement (vécu, discours, écriture, artefact) aussi bien que du savoir lui-même, c'est le néant, parce que lui-seul fait possible la multiplicité. Lyotard désigne ce vide-néant-blanc avec le terme ambigu d'Arakawa - blank. Indéterminé et étrange le blank "est le vide, le néant, où un univers présenté par une phrase explose et s'expose, comme un feu d'artifice, quand advient la phrase, et où il s'éteint avec elle. Cet abîme, le rien qui sépare ces phrases l'une de l'autre, est en outre la "condition" de toute présentation et de toute occurrence, mais cette "condition" $n$ 'est pas saisissable immédiatement par elle-même. Il faut une autre phrase pour tenter de la saisir (...) Le "blank" est la condition de la présentation et de son évanescence, un nom de l'être, peut-être en avance de l'occurrence."1s. En tant que fond ou origine de toute détermination l'indéterminé c'est l'abîme de la liberté origine des différences, raison des antagonismes, où au lieu d'une synthèse universelle ou apperception (Kant) il s'effectue une production imaginative, libre. D'où pour Lyotard chaque consensus n'est qu'une promesse vaine d'universalisation. "Considérez à présent certaines pensées contemporaines relatives à la communication : l'idée d'une unanimité raisonnable que Habermas nomme le Diskurs, la fondation 
ultime de la raison elle-même, que Apel croit trouver dans l'argumentation meta-pragmatique intersubjective; même l'accord librement consenti grâce à la discussion ou à la "conversation" libre, qui serait, selon Rorty, ce qui nous reste de vraiment raisonnable, passé les vains espoirs de "fonder" -, tous ces modèles sont, à un titre ou à un autre, "pragmatistes", tous ignorent le partage du goût, et l'antinomie dont il souffre. Kant explique qu'on ne peut pas argumenter à propos du beau, mais qu'on le doit pourtant. Qu'est-ce à dire? Le caractere essentiel du jugement réflexif où cette absence est portée à son comble (où le concept est à son minimum). Qu'en sera-t-il alors d'une communauté jugeant ainsi et cherchant à valider ses jugements par l'argumentation? Elle sera toujours en train de se faire et de se défaire" ${ }^{116}$.

Cela est, à mon avis, d'autant plus vrai pour les régimes totalitaires qui n'argumentent, mais qui décrètent, ou bien, qui font semblant d'argumenter - les arguments changent mais la réalité reste, puisqu'on peut toujours en trouver une argumentation ou une autre...

Dans sa qualité de post/moderne La condition postmoderne est avant tout une discussion silencieuse avec Kant, une réponse indirecte aux questions : "Que puis-je savoir?" et "Que puis-je espérer?". Pareil à Socrate le penseur postmoderne sait que ce qu'il sait n'est rien et que ce "rien" est tout ${ }^{17}$. Rien - en comparaison au savoir et son certitude apodictique à l'époque de la Raison. Rien - à cause de la conscience même de ce "non" du savoir actuel, où les vérités sont multiples ou in/commensurables. Rien - parce que sa pensée est toujours un différer de "quelque chose". Et il sait, comme l'Etranger de Camus, que ce qu'il "peut" esperrer est encore un "rien" - il est un Différend aussi bien par rapport à la nature que par rapport à la société, créant ses règles de jeu et ses assises. Rien - car Dieu est crucifié. Rien, car ce Différend, ce anti-heros, c'est $\mathbf{K}^{18}$.

\section{Les figures du discours postmoderne}

Si l'on pourrait donc définir le savoir postmoderne en général de facon négative comme prise de conscience de la condition du Différend, la question en quoi consiste le postmodernismeen philosophie paraît bien plus difficile. Non seulement parce qu'il s'agit d'une notion reçue du domaine des arts et de l'architecture, mais aussi parce que, comme l'a bien remarqué Denis Donoghue, le modernisme et le postmodernisme sont des notions aussi confus qu'il semble qu'ils signifient tous ce que nous voudrions qu'ils signifient $t^{19}$. Mais pour ne pas parler de "n'importe quoi" et "n'importe comment" et pour évaluer la place et le rôle du 
postmodernisme il faudrait sinon le définir (ce qui n'est guère possible) du moins délimiter le sens des notions de "postmodernisme", de "postmodernité", de "déconstruction", respectivement de "déconstructivisme". De tentatives pareilles existent déjà, bien que de bases assez différentes, parmi lesquelles les plus sérieux sont peut-être l'analyse généalogique d'Andreas Huyssen et la lecture archéologique de Steven Best et Douglas Kellner ${ }^{20}$. Elles peuvent être divisées en quelques groupes :

1) la définition du postmodernisme comme "ultra" modernisme (Ihab Hassan, Leslie Fiedler) ${ }^{21}$;

2) la considération du postmodernisme comme une rupture ou une "résistance" dans le modernisme, respectivement dans l'art, dans la philosophie, dans l'histoire ou la politique modernes, comme quelque chose qui est "en partie modernisme, en partie quelque chose d'autre" (Charles Jencks, Robert Venturi, Frederic Jameson, Arthur Kroker, David Cook, Andreas Huyssen, Steven Best, Douglas Kellner, Hal Foster) 22 ;

3) description du postmodernisme en établissant ses tendances communes ainsi que les divergences entre ses adeptes (Zigmund Bauman, Gary Madison, Todd Gitlin, John McGovman e.a. $)^{23 ;}$

4) détermination des sens différents de l'usage du concept de postmodernisme (Jonathan Arac, Mike Featherstone e.a.) ${ }^{24}$.

Il existe un autre point de vue, qui n'est pas très répandu mais qui mérite aussi une certaine attention, celui que le postmodernisme n'est qu'une notion inventée parce que ni dans dans les arts tel que p.ex. la peinture, ni dans la philosophie il n'y a pas de courant particulier portant le nom "modernisme" pour qu'on puisse parler de POSTmodernisme.

Il n'est pas difficile de voir les points faibles de ces approches de la notion ambiguë de postmodernisme. Il est clair que dans le premier cas on confond "néo" avec "post"-modernisme et dans le second postmodernisme avec philosophie ou art de la postmodernité, en rendant seulement de façon nouvelle la division bien connue de philosophie ou culture "moderne" et "contemporaine". Dans le troisième cas l'énumération de caractéristiques communes du postmodernisme ou de la pensée postmoderne (comme p.ex. le rejet d'idiomes institutionnalisées, le renoncement aux oppositions traditionnelles, à la mesure de la raison, au sujet absolu, la critique et la déconstruction des totalités et des totalisations, de la sérialité, de la pensée de la présence etc.) mène à la constatation d'éléments de la philosophie et des arts contemporains qui préexistent déjà dans certaines oeuvres modernes ou bien à la confusion de postmodernisme et de déconstructivisme. Dans le quatrième cas on 
propose une description des différentes usages du mot en reproduisant les ambiguités et les contradictions. Le cinquième cas, qui semble assez particulier, s'évanouit aussi quand on accepte de problématiser la notion dans le sens d'Ihab Hassan comme POSTmodernISME ${ }^{25}$.

Mais si sans la délimitation du sens du postmodernisme il est impossible d'en discuter sa relevance méthodologique, le contraire est vrai aussi - le passage à l'analyse terminologique sans l'analyse du paradigme méthodologique du postmodernisme n'est pas possible. C'est pourquoi notre fin ici ne sera ni proprement dénotative, ni descriptive, ni interprétative et encore moins polémique. Il s'agit de faire voir que le postmodernisme est un cas particulier du discours postmoderne et du déconstructivisme afin de concevoir cette relation "commensurable" qui permettrait de fixer les frontieres de leurs significations, aussi bien les champs de validité de leurs prétentions.

On pourrait accepter avec Lyotard que le postmodernisme est une sorte de décentralisation et de déconstruction du sujet absolu et de son discours en tant que fondement du savoir et de la créativité. Mais à ce moment-là le programme de Dada p.ex. s'avérerait comme étant par excellence postmoderniste, tandis que le projet surréaliste de Breton et la méthode paranoïaque et critique de Dali seraient en marge des experiments modernistes. C'est un "fait" que les collages ready-made de Duchamps, en particulier la scandaleuse Joconde L.H.O.O.Q. (1919), devancent plus d'un demi-siècle les estampes "postmodernistes" de Pneumonia Lisa (1982) de Rauschenberg. Mais il n'est pas moins vrai que la célebre "peinture de l'action" de Pollock est née de la confrontation avec le surréalisme et que le sanctuaire de l'architecture postmoderniste - le centre Georges Pompidou - a receuilli les chef-d'oeuvres de l'avant-garde moderniste. Qui pourrait dire aujourd'hui avec exactitude où passe la frontière si fine entre les "grands récits", les récits hyperboliques des héros de la modernité, et les "petits récits" ou les mensonges de ce petit homme "postmoderne" que nous rencontrons comme un passant? Dans La révolte des masses qui continue, peu importe qu'elle soit sociale ou culturelle, les masques du Surhomme sont portés précisément par des gens "sans visages", les petits récits distribuent les plus grands mensonges, tandis que les grands récits sont obligés de vivre les vérités-passions de la prison ou de la clinique.

Il est évident que la définition du postmodernisme comme réaction intellectuelle en face de la "fin des grands récits", comme déconstruction du Sujet et de l'Histoire, n'est pas suffisante et que l'hyperbolisation de ce caractère particulier mènerait à l'impossibilité d'expliquer les bases théoriques de plusieurs de ses représentants les plus 
illustres comme Baudrillard, Rorty, Jameson, Spivak, Kroker e.a. Comment, en effet, pourrait-on expliquer l'union de la méthodologie postmoderniste avec des paradigmes aussi hétérogenes comme le marxisme, le pragmatisme, le structuralisme, l'herméneutique ou le féminisme? Et si l'on devrait faire une différence entre poststructuralisme et postmodernisme, comme l'indique dans ses critiques de Jameson David Shumway ${ }^{26}$, dans quel sens faudrait-il qu'on cherche la différence? Si pour Shumway il s'agit finalement d'une différence dans la problématisation et la critique du "modèle de la profondeur", c-àl-d de l'interprétation, je voudrais démontrer, de mon côté, qu'il s'agit plutôt de deux façons différentes de déconstruire le Sujet et l'Histoire qui ne permettent pas l'identification de postmodernisme et de déconstruction.

On pourrait dire que le commun entre le poststructuralisme de Derrida et le postmodernisme de Lyotard est la mise en probleme de la "fin" ou de la "mort" au sens large; mais alors que dans le premier cas on soumet à la critique la métaphysique de la présence, dans le second on met en question les systèmes et les idéologies de la modernité.

Rappelons qu'un des points culminants dans la métaphysique de la présence, c'est d'après Derrida la phénoménologie husserlienne qui n'est qu'une des formes de la philosophie de la vie puisque l'origine du sens est pour elle toujours définie par l'acte de la vie, échappant à la réduction transcendentale ${ }^{27}$. La grande découverte de la méthode husserlienne grâce à laquelle on se rend compte de la superposition de deux sphéres différentes - psychologique et transcendentale, - c'est le dédoublement de l'époché. C'est par-là précisément qu'apparaît la question de la différence qui ne correspond à aucune dualité ontique ou empirique. Bien que le Je transcendental soit absolument différent du Je psychologique ou de l'homme naturel, la subjectivité reste la même. La nécéssité de définir ce "rien" de la différence de ces sphères parallèles exige, d'après Derrida, une notion nouvelle, "ultra-transcendentale", de la vie, à laquelle on ne pourrait accéder qu'à travers la réinterprétation de la relation entre logos et phoné, démontrant le rôle privilégié de la conscience comme "voix vivante". L'analyse du langage de la phénoménologie démontre en réalité le vice fondamental de la philosophie husserlienne - l'intention de sauvegarder la présence et de réduire ou de déduire le signe ${ }^{28}$. Ce "principe des principes" de la phénoménologie s'exprime dans la certitude que la forme universelle de toute expérience vécue (Erlebnis) et par-là - de chaque vie a été et sera toujours le présent. Dérivé de la présence à soi le sens chez Husserl a un caractère temporel qui est en contradiction avec le fait fondamental que le sens est toujours déjà engagé dans le mouvement de la trace et de la série de 
significations $^{29}$. La signification de la présence n'est pas un sens présignificatif et présuppose donc le manque, l'absence originaire de l'ipséité, c'est-à-dire la differance. Cette différance n'est pas un mot, ni une notion ${ }^{30}$, mais ce qui effectue le différer étant simultanément le dé-poser et le différer de la présence, le co-être de l'arche-écriture menant à la trace grammatologique. La fausse tentative de Husserl de faire dériver la différence de l'actualité de la présence montre en grande partie le vice de la métaphysique occidentale elle-même réduisant le sens au savoir, le logos - à l'objectivité, le langage - à la raison. Il s'agit au fait d'un désir absolu d'entendre son propre langage parlé, $c-a ̀-d$ la voix sans différance, sans écriture qui est à la fois absolument vivante et à la fois absolument morte ${ }^{31}$. Mais l'introduction du co-être et de la trace montre que la signification n'a pas besoin de la présence de la subjectivité, que la valeur significative du Moi ne dépend pas de la vie du sujet parlant et même que notre mort est structurellement nécéssaire pour le prononcement du $\mathrm{Je}^{32}$. Cela révèle aussi le double sens de la crise contemporaine - la séparation du chemin du logos qui se divise en retombant d'une part dans le centrisme de la présence (Husserl) et d'autre part dans le labyrinthe sans issue, $c-a-d$ la décentralisation dans la folie (Foucault). D'après Derrida ces deux chemins se ressemblent du fait qu'ils touchent en réalité à la finitude et à l'ambiguité de la réalité humaine ${ }^{33}$. Et donc ce n'est qu'en considérant l'analogie entre le noumène et l'oromène ${ }^{34}$, en rejettant aussi bien le chemin, que le labyrinthe pour en déceler le fond commun - la différance irréductible de la différence, - qu'on prendra conscience de la monstrueuse hybridité de la véritéts.

Il est évident que la déconstruction de la subjectivité ainsi conçue ne veut pas dire impossibilité, rejet ou même "mort" de son auto-compréhension, du référent un, comme cela est le cas dans certaines variantes extrèmes du postmodernisme et en partie chez Lyotard. Comme l'a fait remarquer Calvin Schrag, cette déconstruction ouvre seulement un espace nouveau, un espace décentré d'interprétation dans lequel on peut superposer les differentes valeurs et approches de la subjectivite ${ }^{36}$. Telle est en particulier l'implication herméneutique de la praxis communicative de Schrag lui-même qui montre que la décentralisation de l'interprétation épistémologique du sujet se mouvant dans l'opposition "intérieur extérieur" leve cette opposition dans l'Ineinander, tandis que la déconstruction de l'enveloppement-dans-le-monde donne la possibilité d'être interprété au moyen de la texture. En ce sens Schrag rejette l'interprétation noncontextuelle de Ricoeur en argumentant que l'existence et la praxis quotidiennes ne sont qu'une texture de la praxis communicative qui 
ne peut pas être réduite ni à la textualité du discours, ni aux tissus de l'activité humaine ${ }^{37}$.

C'est exactement contre de telles positions "réconciliatrices" que Lyotard dirige son projet postmoderniste, qui insiste sur la discontinuité et l'hétérogénéité des discours contemporains. Déjà en Discours, figure il accentue, non sans raison, sur l'irréductibilité de l'espace figuratif de l'imagination à l'ordre discursif. En dérivant sa puissance des figures de l'inconscient, l'art interrompt la concorde prétendue de la conformité et de la communication ce qui met le règlement logique sur l'arrière-plan ${ }^{38}$. Ce point de vue a des conséquences importantes non seulement pour l'art, mais aussi pour la theorie sociale et politique. Ainsi, si les systemes et les idéologies de la modernité n'ont été que des métadiscours, légitimant le savoir et le pouvoir, respectivement la "justice commune", la condition postmoderne est la révélation de l'échec de l'universalisme et des totalisations montrant, d'une part, l'atomisation de la société et, d'autre part, le passage du pouvoir d'état dans les mains d'une administration de plus en plus dépersonnalisée et emprisonnée par la technocratie. Cet effacement de l'individualisme, des institutions et des traditions mène à une crise d'identification où chaqun est renvoyé à soi, mais ce "soi" est trop impuissant, "pris dans une texture de relations plus complexe et plus mobile que jamais"39. Par-là se pose la question centrale du Différend : comment deux phrases hétérogènes peuvent-elles se rapporter à un même objet, respectivement qui est le sujet de la légitimation? Comment est-il possible de démontrer rationnellement la monstruosité des chambres à gaz puisqu'on ne peut pas faire venir les morts pour qu'ils racontent leurs souffrances? Cette impossibilité de démontrerl'injustice fait de l'offensé une double victime. Mais les figures d'hétérogenéité témoignent de quelque chose bien plus important que l'impossibilité de donner des preuves, de fournir une interprétation ou compréhension de soi; ils témoignent surtout que pour la plus grande partie le soi-disant "règlement" des conflits ne règle rien, puisqu'il ne fait qu'imposer l'une des idiomes à l'autre ce qui prouve encore une fois l'irréductibilité du singulier et des particularités ${ }^{40}$.

Contre le scandale intellectuel de la modernité qui consiste d'après Claude Lanzmann dans la tentative d'interprétation historique "comme s'il pourrait y avoir une genèse harmonique de la mort" ${ }^{41}$ se déclareraient tous les penseurs postmodernes. Bien qu'ils reconnaissent une certaine validité de l'interprétation et même certaines formes de "totalisation", basées sur les homologies et la traduction des codes qui peuvent mener à l'effet de la vérité", Baudrillard, Jameson et Kroker expriment la tendance menant à la dissolution de l'histoire. D'après 
Baudrillard la logique du confort a abouti dans le capitalisme à la formation d'un système d'autoréférence tout à fait accidentel. La culture de consommation est devenue distributrice d'images et de signifiés d'une société de simulacres et de simulation, qui a effacé la différence entre le réel et l'imaginaire aboutissant à l'hallucination de la réalité, privée de toute profondeur. Cette découverte du nihilisme au coeur de la logique capitaliste du comfort vise la destruction des "illusions référentielles" - les totalités sociales privilégiées comme le travail, la valeur de consommation, la science, la société, le sexe, l'émancipation etc., et leurs théories, que Lyotard appelle métadiscours, sont immergées dans le brouillard des simulacres. Cette disparition de l'histoire dans la désintégration des liens sociaux transforme la société en une masse amorphe d'individus isolés ${ }^{42}$. Bien qu'il s'oppose à une robinsonnadequi serait le résidu nostalgique de l'idée paradisiaque d'une société organique perdue, Lyotard, pour qui les liens sociaux sont des expressions langagieres pris dans la texture complexe du discours, aboutit à la reconnaissance de la dissémination des jeux de langage et par-là - à la dispersion du sujet social ${ }^{42}$. Encore plus radical à cette occasion semble Jameson pour qui la différence stylistique et l'hétérogenité des pastiches et des simulacres ne sont que des témoignages de la perte du référent et et de la "mort du sujet". La fragmentation des discours est l'effet de la fragmentation de la temporalité et du sens de l'histoire dans le paradigme social qui est fondamental pour Jameson et Baudrillard - la schizophrénie ${ }^{43}$.

Il est évident que les déconstructions postmoderniste et poststructuraliste du Sujet et de l'Histoire sont basés sur deux conceptions de la "mort" ou de la "fin" bien differentes, d'où les différences de leur fin et de leur signification. Si la déconstruction postmoderniste est fondée sur la "mort" du sujet et de l'histoire comme dissolution, dépersonnalisation et effacement et vise la destruction de l'illusion du référent un communement valide, respectivement de la possibilité d'une explication épistémologiquement légitime et valide, la déconstruction poststructuraliste insiste sur la "mort" comme un moment indispensable de la prise de conscience de l'origine impersonnelle de la subjectivité et de l'histoire (moment qui est présent aussi bien chez Derrida, que chez Lévi-Strauss et Foucault), qui ne sont pas rejetés mais problématisés à travers l'impossibilité d'être extraits des discours particuliers. Le poststructuralisme ne nie pas le référent ou le signifé, mais démontre son insuffisance et par-là - la nécéssité de passer à des couches d'interprétation plus profondes. Son projet déconstructif n'est pas destructif, comme le croient beaucoup de ses opponents, mais critique du fait que son but final n'est pas la destruction de l'interprétation philosophique, 
sociale et historique, mais la décomposition de son unanimité. Plus radical dans son programme le postmodernisme est un cas particulier du discours postmoderne aussi bien que du déconstruc-tivisme - son expression extreme ou "ultra-déconstructivisme".

De ce point de vue Dada et le surréalisme acquériraient un statut nouveau. Le "rien" proclamé dans le Manifeste de Dada serait le fond postmoderne de la subjectivité, tandis que la révolution surréaliste - une destruction postmoderniste des simulacres sociaux. Mais ce statut n'a rien de définitif: Dada, le surréalisme et tous les autres courants intellectuels ou artistiques de la postmodernité pourraient se prêter à une lecture postmoderne aussi bien qu'à une lecture postmoderniste. Ce qui est définitif par contre, ce sont les conclusions qui s'ensuivent de la différence mentionnée, $c-a-d$ que si le discours postmoderne pourrait servir à l'explication du postmodernisme, le cas contraire est impossible. Même quand il se met à dis-courir la modernité le postmodernisme échoue car il se localise et dissipe dans la texture désagrégée et parce que par son point de départ même son discours ou récit est régional. Fixé sur les images d'une "hyperréalité" dont les signes ne sont ni vrais, ni faux, le postmodernisme n'est pas tellement une explication de la postmodernité mais plutôt sa passion mortelle ou bien son défi mérité.

\section{Jeux (non) partages}

Bien que La condition postmoderne a été souvent interprété comme une rupture dans la tradition elle n'est pas moins son réinterprétation. Si nous nous limitons à la tradition française nous ne pourrons pas ne pas entrevoir les lectures de Sartre ou de Foucault et par-là - la lecture critique de Descartes. Car si l'être humain n'est pas ce qu'il est, puisqu'il est ce qu'il n'est pas (Sartre), cela veut dire qu'il porte en soi dès le début la différence, c'est-à-dire la puissance de différer et de différencier cọme caractère ontico-ontologique. Cela veut dire aussi qu'il n'y a pas de modele stable de l'homme, que son accord impossible est une identité, respectivement une identification impossible. Ou bien, comme consta-tera Foucault, il y a des êtres humains et non pas $d^{\prime \prime}$ homments. L'homme est une invention de la modernité sans consistance car l'être humain réel ne peut être analysé ni comme objet (analyse scientifique), ni comme sujet (totalisation philosophique). La formule rationnaliste de la modernité "Cogito ergo sum" n'est plus applicable aux conditions contemporaines où la pensée n'est plus une forme commune de l'existence du concret, mais émancipation des apparitions individuelles qui décomposent l'universel et se caractérisent le plus souvent par les 
déviations ${ }^{46}$.

Il est évident que la position anti-cartésienne de Foucault ne reproduit pas simplement l'antinomie "abstrait-concret"dont Kierkegaard s'en sert pour sa critique de Hegel. L'impossibilité de faire dériver l'existence humaine de la pensée prouve non seulement l'échec de la philosophie moderne, mais aussi un fait beaucoup plus fondamental- le changement de la situation contemporaine qui s'exprime dans la limitation du normal et du rationnel par l'inconscient et le pathologique. Aussi extrème qu'il peut paraître ce point de vue n'est pas typique seulement pour Foucault. Des constatations comme : "l'époque est une vérité, mais une vérité qui s'ignore" (Sartre) ${ }^{47}$, "les hommes font leur histoire, mais ne le savent pas" (Levi-Strauss) ${ }^{48}$, "non seulement l'homme ne pourrait être compris sans la folie, mais il ne serait pas homme s'il ne la comportait comme limite de sa liberté" (Lacan) ${ }^{49}$, "le libido doit pénétrer le champ social sous ses formes inconscientes et par-là halluciner l'histoire, mener au délire les civilisations, les continents, les races" afin de détruire l'inconscient Oedipien répressif et de le remplacer par l'inconscient immédiatement productif (Deleuze, Guattari) ${ }^{50}$ etc., deviennent une praxis qui détruit tout ce qui est devenu dogme dans des sphères problématiques en commençant par la théorie et en aboutissant à la politique (Derrida).

De même chez Lyotard le Cogito est déconstruit afin d'acceder à l'élément fondamental de la figuration pure ou "figure-matrice" qui est le désir qui nous met en face de l'activité schizophrénique productrice et sa révolution permanente ${ }^{51}$.Pourtant la musique libidinale dont parle Lyotard n'est pas une recherche de la folie car cela aurait pour effet encore la légitimation du pouvoir des normes : "les despotes ont besoin de leurs fous : leur justification, la représentation en cours de ce qui est exclu. Comme les médecins de leurs malades et les politiques de leurs ouvriers"52. Lyotard nie de même l'opposition discursive continu/discontinu en tant que dérive logique incapable d'expliquer le changement dans la situation du savoir actuel. Et pourtant les effets de cette déconstruction, les cheminements du savoir postmoderne qui esquissent son "labyrinthe", sont clairs et logiques - refus des règles universelles, mise en question de l'identification socio-politique et culturelle, problématisation de la praxis communicative et des institutions. Bref, si les métarécits ont perdu leur légitimité, celle-ci devrait être cherchée ailleurs - dans les récits eux-mêmes, $c-\grave{a}-d$ dans le local et non pas dans l'universel.

Ce remplacement de la formule hegelienne "la totalité est tout" avec "les éléments concrets sont tout" n'est pas nouveau. Depuis les 
débats du Moyen-Age entre nominalistes et réalistes jusqu'à la discussion phénomenologique comment faut-il entendre l'appel " $Z u$ den Sachen selbst!" la philosophie a eu bien de polémiques de ce genre qui ont mis en question non seulement le savoir, mais aussi ses propres limites. S'il y a quelque chose qui étonne dans ce cas, c'est que ni Nietzsche, ni Kierkegaard, ni le dernier Husserl n'ont pas provoqué tant de commentaires critiques que Lyotard. Commentaires qui varient entre le rejet radical de sa pensée jusqu'à sa réception partielle en passant par la supposition qu'il pourrait avoir raison, mais alors tant pis pour l'humanité...

La célébrité de Lyotard est due sans doute à ses critiques et surtout à sa grande polémique avec Habermas. Il est à remarquer que l'apologète aussi bien que le criticien de la modernité partent des mêmes origines tout en voyant une même mission pour la philosophie - la dénonciation des idéologies. En fondant comme les poststructuralistes et les postmodernistes le savoir dans le désir, dans l'intérét Habermas effectue une archéologie particulière qui à la différence de celle de Foucault ne vise pas la découverte des structures discontinues, mais l'esquisse de la continuité de la réflexion universelle au moyen des contradictions réelles des intérêts ${ }^{53}$. Il est donc évident que ces deux dérives à partir de Marx et Freud partent d'un même fondement pour arriver à deux perspectives opposées.

La théorie moderne de la société, comme le constate Lyotard, est partagée entre deux modèles - l'un qui présente la société comme un tout fonctionnel et organique (Comte, Parsons, Luhmann) et l'autre qui considère la société comme divisée en deux (marxisme, théorie critique) ${ }^{54}$. Le second modèle auquel se rattache l'alternative de Habermas présente la société comme divisée et donc comme ayant besoin d'être réunie. Son programme épistémique est "critiquen", dirigé contre le fonctionnalisme, contre la mise du savoir au service du pouvoir. Le savoir doit aider l'autoformation de l'humanité, le dépassement de ses antagonismes et la création d'une nouvelle société commune au moyen du dialogue des argumentations diverses visant le consensus, $\mathrm{c}-\mathrm{a}-\mathrm{d}$ d au moyen du Diskurs. Bien qu'il prend en considération le moment positif d'une telle position - la récupération de l'autonomie du savoir et son dépouillement des utilisations technocratiques et politiques - Lyotard souligne que "la cause est bonne, mais les arguments ne le sont pas ${ }^{n s 5}$. Car il s'en suit que le but final est l'aboutissement à tout prix au consensus et non pas à la justice, la poursuite du commun, de l'isomorphisme des jeux de langage qui n'existe pas et par-là - le recours à la terreur. Ce que Habermas et ses éleves répliquent à de telles critiques, c'est que l'argumentation comme 
remplissement de l'exigence de validité et l'acceptation par le consensus de quelque chose pour vrai et légitime ne signifie pas forcer quelqu'un à croire une chose ou une autre, ne signifie pas d'imposer à l'autre notre propre interprétation; il s'agit, tout au contraire, de chercher des procédures communement valides pour résoudre les conflits provoqués par les antagonismes des intérêts et des positions ${ }^{56}$. La critique générale de Habermas contre les discours postmodernistes consiste donc dans l'objection qu'ils ignorent le lien profond entre la rationalité moderne et la modernisation de la société contemporaine et qu'ils les relativisent, qu'ils n'ont pas de modele d'explication et refusent tout approche théorique ${ }^{57}$. Cette critique adressée contre Lyotard est articulée en particulier par Axel Honneth ${ }^{58}$, Douglas Kellner ${ }^{59}$, Nancy Fraser, Linda Nicholson et Seyla Benhabib ${ }^{60}$.

Il est symptomatique que non seulement les adeptes de la praxis communicative et de l'éthique discursive, mais aussi les représentants et sympathisants du postmodernisme ont des réserves critiques contre les implications sociales de La condition postmoderne. Un cas assez typique en ce sens est Rorty qui a pris, comme on le sait, une position intermédiaire entre Habermas et Lyotard. D'après Rorty Lyotard est prêt de rejeter la politique libérale afin d'éviter la philosophie universaliste alors que Habermas essaie de trouver un appui dans la philosophie universaliste pour donner son soutien à la politique libérale. Le reproche fondamental de Rorty contre Lyotard, c'est que le dernier refuse de s'identifier avec un contexte social quelconque ${ }^{61}$. Pour Rorty le détachement de la philosophie de la réforme sociale est une possibilité de remettre en question la tradition, mais ce n'est pas la seule. Une autre serait de retrouver le sens de cette tradition pour créer un canon nouveau d'après lequel la marque spécifique du "grand philosophe" serait la prise de conscience des nouvelles possibilites sociales, religieuses et institutionnelles telles que la démocratie et la open society. C'est en cela que consiste le chemin intermédiaire entre Habermas et Lyotard. Pour Rorty l'éloge de la démocratie parlementaire des Etats-Unis et du welfare state est fondée, car elle a ses raisons dans la comparaison concrete avec les autres alternatives existantes et non dans les métanarratives légitimant ces institutions comme étant plus adéquates à l'eternelle nature humaine ou aux lois morales universelles. Pareil à Lyotard Rorty rejette l'approche métaphysique du développement social, mais à la différence de lui il est convaincu que le changement social doit s'effectuer à l'aide de la "fantaisie utopique", visant la constitution d'un milieu où chacun aura la possibilité de proposer des voies pour l'établissement d'un monde (galactique) commun et où toutes ces propositions 
seront librement discutées. En réponse à Lyotard qui lui reproche l'éthnocentrisme proche à la terreur nazi Rorty répond qu'il y a une difference entre les nazis qui disent 'nous sommes bons, parce que nous sommes un groupe particulier' et les réformistes liberaux qui disent 'nous sommes bons parce que par conviction et non par force nous arriverons à convaincre les autres que nous sommes bons" ${ }^{362}$. Aucun événement historique, même Auschwitz, ne pourrait remettre en question l'ordre de la démocratie libérale et son utopie cosmopolitique, si ce n'est une conception meilleure de l'organisation de la société, $c-\hat{a}-d$ une utopie plus convaincante ${ }^{63}$.

Or ce que Rorty et les autres opposants de Lyotard ne prennent pas en considération, c'est en premier lieu le fait que l'auteur de $\mathrm{La}$ condition postmoderne ne nie pas la philosophie, ni la théorie y compris la theorie sociale, mais qu'il exige, au contraire, qu'elles soient "ouvertes" pareil à la "systématique ouverte" de Salanski. Il souligne en ce sens que toute action a besoin de règles, c-à-d de métapréscriptifs, et que "l'activité différenciante, ou d'imagination, ou de paralogie dans la pragmatique actuelle, a pour fonction de faire apparaître ces métapréscriptifs (les "présupposés"), et de demander que les partenaires en acceptent d'autres ${ }^{n 64}$. Cela signifie qu'il faut reproduire l'hétérogénéité dans les discours philosophiqueset socio-politiqueseux-mêmes, qu'il faut analyser les divers jeux de langage dans leur conflictualité et non de les "réduire" à une alternative globale telle p.ex. la démocratie libérale de type américain, seulement parce qu'elle est le meilleur des mondes possibles. Meilleur pour qui...?

Deuxièmement, le refus de lier la rationalité directement à la théorie sociale (refus qui est caractéristique non seulement pour Lyotard mais aussi pour des penseurs comme Philippe Lacou-Labarthe et Jean-Luc Nancy qui ont lancé le programme du retrait du politique ${ }^{65}$ ), ainsi que la fuite intellectuelle de l'identification sociale n'est pas forcément un vice, mais plutôt un acte justifié historiquement et intellectuellement rejetant la réduction de la philosophieà la légitimation d'une ligne politique ou de parti afin de conserver sa fonction de critique authentique.

Et, finalement, si Lyotard insiste sur le fait que la voie pour atteindre la justice sociale n'est pas le consensus universel et à tout prix, ni dans la validité communément reconnue, cela ne veut pas dire qu'il exclut toute possibilité de dialogue, de consensus et d'argumentation. Mais comment ne pas rappeler que le consensus ainsi dit a trop souvent amené à des résultats injustes comme la domination d'un groupe par un autre et la position d'un règlement pris pour valide afin de garantir les 
interêts d'un des partis? Ainsi la terreur se reproduit-elle toujours et toujours - une personne ou un groupe est exclu ou condamné d'un autre groupe avec consensus. C'est pourquoi Lyotard insiste dans ses Moralités postmodernes: "On nous dit que les messages s'échangent à condition qu'ils soient comprehensibles... et à condition que vous et moi puissions occuper tour à tour les positions du locuteur et de l'allocutaire. Richard Rorty va jusqu'à soutenir que cette condition pragmatique suffit, à elle seule, à garantir la solidarité démocratique, sans considération de ce qui se dit ni de la manière de le dire. La langue peut être "blanche", comme celle de l'Etranger de Camus, il n'importe que de l'adresser à autrui. Les langues humaines conferent structurellement au locuteur la capacité de parler aux autres. Mais capacité n'est pas devoir. On n'a encore jamais prouvé qu'un silence voulu soit une faute. Ce qui est un crime, c'est de l'imposer à l'autre. On exclut celui-ci de la communauté interlocutrice et, de surcroît, on ajoute à ce dommage un tort plus grave encore, puisque interdit de parole, le banni n'a pas les moyens de faire appel de son bannissement. Politique, social ou culturel, tel est l'exercice de la terreur: priver l'autre du pouvoir de répliquer à cette privation. Quoi qu'on pense, la peine de mort, si légale soit-elle, évoque toujours ce crime. Mais, aussi bien, l'enfant à qui ses camarades disent qu'ils ne joueront pas avec lui et que ça ne se discute pas est en vérité victime d'un crime contre l'humanité $e^{n 6}$. Ainsi la réalité crée chaque jour le différend et la question n'est pas de chercher simplement à dépasser la discorde par un nouvel accord, mais de chercher des voies qui puissent limiter ces crimes, cette exclusion et agression contre l'Autre.

Cette idée est explorée surtout par le "difference feminism", qui insiste sur la nécéssité d'entendre aussi l'autre voix ${ }^{67}$, et la "politique de la différence" qui tout en rendant compte des antagonismes des groupes essaie d'entrevoir les possibilités de dépasser la domination pour accéder à la justice et au respect mutuel. "Les relations entre les identités culturelles et de groupe dans notre société - souligne p. ex. Iris Youngsont pleines de racisme, de sexisme, de xénophobie, d'homophobie, de soupçon et de mépris. La politique de la difference rejette la pensée institutionelle et idéologique pour reconnâtre de deux façons fondamentales les groupes différemment identifiés : au moyen de la représentation politique des intérêts de groupe et au moyen de l'appel au caractéristiques culturelles particulières des groupes différents" ${ }^{168}$.

L'approche de Lyotard pourrait être appliqué de façon semblable à l'interprétation et à la solution de "la crise de la philosophie" ainsi-dite. Car si la philosophie voudrait sortir de la non-philosophie, il faut qu'elle se constitue non pas comme une philosophia perennis vouée à fournir des 
clés éternelles et universelles, mais comme une herméneutique des differences, comme un jeu de discours philosophiques multiples dont le devenir polyphonique n'est que le devenir de la philosophie en tant que telle. La culture philosophique ne serait donc plus l'Aufhebung des positions anti-thétiques dans la notion generale de philosophie, mais l'en-je(u) de ces positions. Et de ce fait-là précisément l'Aufhebung hegelienne elle-même ne serait plus le fait d'une généralité mais celui du particulier - une forme ou jeu de langage possible parmi d'autres possibilités de la pensée discursive dont il constitue la différence. A ce moment-là préférer un discours philosophique plutôt qu'un autre, c'est justement une question de préférence ou de choix et non pas de vérité. La vérité, c'est qu'il y a des vérités particulières qu'aucun discours ne pourrait rendre, si ce n'est celui de la Voix et de la Révélation. En ce sens l'inaccessible je(u) des jeu(x) de Lyotard serait le je(u) de la Voix imprononçable, ce je(u) tout autre et tout différent de nous-invisible, intemporel, dont l'historicité n'est que la mort. Mystère des mystères qui ne se manifeste à nous qu'à travers sa lettre déposée, la Tora, et le Fils, qui est l'Alliance: "Vous n'entendrezl'incarnation que si la Voix incarnée vous parle, parle par vous en vous" ${ }^{\text {} 69}$.

C'est sur cette pensée de la différence, qui doit renforcer notre capacité de supporter l'incommensurable ${ }^{70}$, que se joue toute la danse philosophique du "post"moderne et du postmodernisme. Danse - libérée des canons du classique, mais qui est provoquée par lui et qui s'en nourrit. Danse mystique - qui échappe aux normes instituées vers les ténébres de l'inconnu. Danse - qui met l'Autre avant le Même pour rejeter la tradition de répression et de domination (Lévinas) ${ }^{71}$. Danse qui ne vient pas des pas ni des règles mais du jeu lui-même. Ou comme dirait Derrida: "La 'vérité' de l'utile n'est pas utile, la 'vérité' du produit n'est pas un produit. La vérité du produit 'chaussure' n'est pas une chaussure .- Mais on pourrait penser la différence de l'être à l'étant comme la chaussure, à travers elle, dans son pas. Et ainsi la différence ontologique: chaussée en peinture ${ }^{\text {772 }}$.

Reste la question pourquoi cette danse de "je est un autre" ${ }^{n 73}$ est la plus solitaire de tous.

Voila la farce.

Institute for Philosophical Research, Bulgarian Academy of Sciences

Yvanka Raynova 


\section{BIBLIOGRAPHIE}

1J.-F. Lyotard. Economie libidinale. Paris: Minuit, 1974, p. 28.

${ }^{2}$ Don Ihde. Postphenomenology. Essays in the Postmodern Context. Evanston, Illinois: Northwestern University Press, 1993, p. 150.

${ }^{3}$ Cf.: A. Huyssen. Mapping the Postmodern. In: New German Critique, Fall 1984, vol. 33, p. 52.

${ }^{4} \mathrm{Cf}$. S. Kierkegaard. Post-Scriptum aux miettes philosophiques. Paris: Gallimard, 1941, p. 240.

5J.-F. Lyotard. La condition postmoderne. Paris: Minuit, 1979, p. 67.

${ }^{6}$ Cf. G. Deleuze. Nietzsche et la philosophie. Paris: PUF, 1962, p. 106.

7J.-F. Lyotard. Economie libidinale. Paris: Minuit, 1974, p. 9.

${ }^{8} \mathrm{~F}$. Nietzsche. Der Wille zur Macht. In: Werke. Auswahl in zwei Bänden.

Bd. II, Stuttgart: Kröner, 1940, S. 422.

Ibidem., p. 424.

${ }^{10}$ J.-F. Lyotard. La condition postmoderne. p. 67.

${ }^{11}$ Ibidem., p. 65.

12J.-F. Lyotard. Instructions païennes. Paris: Gallilee, 1977.

13J.-F. Lyotard, J.-L. Thébaud. Au juste. Paris: Bourgeois, 1979, p. 81; cf. Rudiments païens. Paris: 10/18, 1973.

${ }^{14}$ Cf. J.-F. Lyotard. Perégrinations. Paris: Galilee, 1990, p. 42.

${ }^{15} \mathrm{~J} .-\mathrm{F}$. Lyotard. Pérégrinations. p. 67.

${ }^{16}$ Ibidem., p. 77.

${ }^{17}$ "Un rien qui est tout" (J.-F. Lyotard. Economie libidinale. Paris: Minuit, 1974, p. 302).

${ }^{18} \mathrm{Cf}$. G. Deleuze, F. Guattari. Kafka. Pour une litterature mineure. Paris: Minuit, 1975.

${ }^{19} \mathrm{Cf}$. D. Donoghue. The Promiscuous Cool of Postmodernism.- New York Times Book Review, June 22, 1986, p.1.

${ }^{20} \mathrm{Cf} .:$ A Huyssen. Mapping the Postmodern. In: New German Critique, Fall 1984, vol. 33, pp. 5-52; After the Great Divide: Modernism, Mass Culture, Postmodernism. Indiana University Press, Bloomington, 1986; Steven Best, Douglas Kellner. Postmodern Theory. New York: The Guilford Press, 1991.

${ }^{21} \mathrm{Cf}$. I. Hassan. The Dismemberment of Orpheus: Toward a Postmodern Literature. Madison: University of Wisconsin Press, 1971 (1982); Paracriticism: Seven Speculations of the Times. The University of Illinois Press, Urbana, 1975, pp. 39-59, The Culture of Postmodernism.- Theory, Culture \& Society, 1985, 2 (3), pp. 119-132.

${ }^{22} \mathrm{Cf}$. Ch. Jencks. The Language of Post-Modern Architecture. New York: 
Pantheon, 1977; R. Venturi, et a. Learning from Las Vegas: The Forgotten Symbolism of Architectural Form. MIT Press, Cambridge, 1977; F. Jameson. The Political Unconscious: Narrative as a Socially Symbolic Act. Cornell University Press, Ithaca, New York, 1981; Postmodernism, or The Cultural Logic of Late Capitalism.- New Left Review, 1984, no. 146, pp. 52-92; Regarding Postmodernism - A Conversation with Frederic Jameson.- Social Text, 1987, no.17, pp. 29-54; A. Kroker. Baudrillard's Marx.- Theory, Culture \& Society, 1985, 2 (3), pp. 69-84; A. Kroker, D. Cook. The Postmodern Scene: Excremental Culture and Hyper-Aesthetics. St. Martin's Press, New York, 1986; A. Huyssen. op. cit.; Steven Best, Douglas Kellner. Postmodern Theory. The Guilford Press, NY, 1991, pp. 1-5; The Anti-Aesthetic.(Hal Foster ed.) Bay Press, Washington, 1983.

${ }^{23}$ Cf. Z. Bauman. On the Origins of Civilisation; A Historical Note.Theory, Culture \& Society, 1985, 2 (3), pp. 7-14; G. Madison. The Hermeneutics of Postmodernity. Bloomington and Indianapolis: Indiana University Press, 1988, pp. IX-XV and 61; T. Gitlin. Life in the Postmodern World (en bulgare).- Spectrum, 1991, no. 72, pp. 12-18; John McGovman. Postmodernism and its Critics. Ithaca and London: Cornell University Press, 1991.

${ }^{24} \mathrm{Cf}$. J. Arac. Introduction to Postmodernism and Politics.- Theory and History of Literature. Vol. 28, Minneapolis: University of Minnesota Press, 1986, pp. IX-XXXIX; M. Featherstone. In Pursuit of the Postmodern.- Theory, Culture \& Society, 1988, 5 (2-3), pp. 195-215; Postmodernism, Cultural Change, and Social Practice.- Postmodernism/Jameson/Critique. Washington: Maisonneuve Press, 1989, pp. 117-138.

${ }^{25} \mathrm{Cf}$. I. Hassan. The Dismemberment of Orpheus..., pp. 259-271.

${ }^{26} \mathrm{D}$. Shumway. Jameson/Hermeneutics/Postmodernism.-Postmodernism/ Jameson/Critique. Washington: Maisonneuve Press, 1989, pp. 198-200

${ }^{27} \mathrm{~J}$. Derrida. La voix et le phénomène. Introduction au problème du signe dans la phénoménologie de Husserl. Paris: PUF, 1967, pp. 7 et 14

${ }^{28}$ Ibidem., p. 57.

${ }^{29}$ Ibidem., p. 96.

${ }^{30}$ J. Derrida. Marges de la philosophie. Paris: Minuit, 1967, p. 7.

${ }^{31} \mathrm{~J}$. Derrida. La voix et le phenomène, p. 115.

${ }^{32}$ Ibidem., p. 107-108.

${ }^{33}$ J. Derrida. L'ecriture et la différence. Seuil, Paris, 1967, p. 97. 
34Cf. J. Derrida. La dissémination. Seuil, Paris, 1972, p. 93.

${ }^{35} \mathrm{~J}$. Derrida. L'écriture et la différence, p. 428.

${ }^{36} \mathrm{C}$. O. Schrag. Communicative Praxis and the Space of Subjectivity. Bloomington: Indiana University Press, 1989, p. 120-121.

${ }^{37}$ Ibid., p. 171.

${ }^{38}$ J.-F. Lyotard. Discours, figure. Paris: Klincksieck, 1971, p. 17.

${ }^{39}$ J.-F. Lyotard. La condition postmoderne, p. 30-31.

${ }^{40}$ J.-F. Lyotard. Le différend. Paris: Minuit, 1983, pp. 215- 230.

${ }^{41} \mathrm{C}$. Lanzmann. L'amour et la haine.- La nouvelle revue de psychanalyse, 1986, no. 33, p. 13.

${ }^{42} \mathrm{Cf}$. J. Baudrillard. Pour une critique de Péconomie politique du signe. Paris: Gallimard, 1972, pp. 31- 48; Le Miroir de la production. Paris: Casterman, 1973, p. 103-115; A Pombre des majorites silencieuses, ou la fin du social. Paris: Utopie, 1978, p. 128.

${ }^{43}$ J.-F. Lyotard. La condition postmoderne, pp. 31 et 66.

${ }^{44} \mathrm{Cf}$. F. Jameson. Postmodernism and the Consumer Society.- The AntiAesthetic. Port Townsend: Bay Press, 1983, pp. 115-119,

Baudrillard. A Pombre des majorites silencieuses..., p. 79.

${ }^{45}$ M. Foucault. Les mots et les choses. Paris: Gallimard, 1966, p. 333.

${ }^{46}$ Ibidem., p. 334-336.

${ }^{47}$ J.-P. Sartre. Vérité et existence. Paris: Gallimard, 1989, p. 131.

${ }^{48}$ C. Lévi-Strauss. Anthropologie structurale. Paris: Plon, 1958, p. 31.

${ }^{49}$ J. Lacan. Ecrits. Paris: Seuil, 1966, p. 575.

50J. Deleuze, F. Guattari. Capitalisme et schizophrénie. L'Anti-Oedipe. Paris: Minuit, 1972, p. 117.

${ }^{51}$ J.-F. Lyotard. Discours, figure. Paris: Klincksieck, 1971, p. 326.

52J.-F. Lyotard. Economie libidinale. Paris: Minuit, 1974, p. 309.

${ }^{53} \mathrm{Cf.:}$ J. Habermas. Erkentnis und Interesse. Frankfurt am Main: Suhrkamp, 1968.

54J.-F. Lyotard. La condition postmoderne. p. 24.

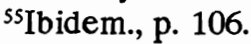

${ }^{56} \mathrm{~J}$. Habermas. Theorie des kommunikativen Handelns. Bd. I, Frankfurt am Main, 1981, S. 20 ff.; Der Philosophische Diskurs der Moderne. Frankfurt am Main, 1985, S. 396 ff.; W. Welsch. Unsere postmoderne Moderne. Weinheim, 1987, S. 69; S. Benhabib. Epistemologies of Postmodernism: A Rejoinder to Jean-François Lyotard. In: Feminism/Postmodernism, New York and London: Routledge, 1990, p. 114.

${ }^{57}$ J. Habermas. Der Philosophische Diskurs der Moderne, S. 3. The Entwinement of Myth and Enlightenment: Rereading "Dialectic of Enlightenment". In: New German Critique, 1986, No. 26, p. 28. 
${ }^{58}$ Ibidem., p. 155.

${ }^{59} \mathrm{Cf} .:$ D. Kellner. Postmodernism as Social Theory: Some Challenges and and Problems. In: Theory, Culture \& Society, 1988, No. 5, pp. 239-264.

${ }^{60} \mathrm{Cf} .:$ Feminism/Postmodernism. New York and London: Routledge, 1990, pp. 34, 125.

${ }^{61}$ R. Rorty. Essays on Heidegger and others. Cambridge: Cambridge University Press, 1971, p. 174.

${ }^{62} \mathrm{R}$. Rorty. Objectivism, relativism, and truth. Cambridge: Cambridge University Press, 1991, p. 214.

${ }^{63} \mathrm{Ibidem}, \mathrm{p} .220$.

${ }^{64} \mathrm{~J} .-\mathrm{F}$. Lyotard. La condition postmoderne. p. 105.

${ }^{65} \mathrm{Cf}$.: Ph. Lacou-Labarthe, J.-L. Nancy. Les fins de $\mathrm{P}$ homme: $A$ partir $d u$ travail de Jacques Derrida. Paris, 1981, pp. 493-497.

${ }^{66} \mathrm{~J} .-\mathrm{F}$. Lyotard. Moralites postmodernes. Paris: Galilee, 1993, p. 179.

${ }^{67} \mathrm{Cf}$.: C. Gilligan. In a different Voice. Cambridge: Harvard University Press, 1982; E.F.Kittay, D. T. Mayers (eds.). Women and Moral Theory. Totowa, 1987; S. K. White (ed.). Lifeworld and Politics: Between Modernity and Postmodernity. University of Notre Dame Press, 1989; S. K. White. Political Theory and Postmodern Problematic. Cambridge University Press, 1991.

${ }^{68}$ I. Young. The Ideal of Community and the Politics of Difference. In: Feminism/Postmodernism. Routledge, 1990, p. 319.

${ }^{69} \mathrm{~J} .-\mathrm{F}$. Lyotard. Un Trait d'union. Québec: Le Griffon d'argile, 1993, p. 39.

${ }^{70 J}$ J.F. Lyotard. La condition postmoderne. p. 9.

${ }^{71} \mathrm{Cf}$.: E. Levinas. La philosophie et Iidée de PInfini. In: A. Peperzak. On the Other. West Lafayette: Purdue University Press, 1993, pp.7980.

${ }^{72}$ J. Derrida. Restitutions de la vérité en peinture.- Macula, 1978, No.1-2, p. $11 \mathrm{ff}$.

${ }^{73} \mathrm{Je}$ est un autre (Rimbaud). 